\title{
Identification of a Novel Transcription Factor Required for Osteogenic Differentiation of Mesenchymal Stem Cells
}

\author{
Francesca Querques, ${ }^{1,2,{ }^{*}, \dagger}$ Anna D’Agostino, ${ }^{1,3, \dagger}$ Carmine Cozzolino, ${ }^{1,2}$ Luca Cozzuto, ${ }^{4}$ \\ Barbara Lombardo, ${ }^{1,2}$ Eleonora Leggiero, ${ }^{1}$ Carlo Ruosi, ${ }^{5}$ and Lucio Pastore ${ }^{1,2}$
}

Osteogenic differentiation is a complex and still poorly understood biological process regulated by intrinsic cellular signals and extrinsic microenvironmental cues. Following appropriate stimuli, mesenchymal stem cells (MSCs) differentiate into osteoblasts through a tightly regulated multistep process driven by several transcription factors and characterized by the expression of a number of bone-specific proteins. In this study, we describe a novel transcription factor that we named osteoblast inducer (ObI)-1, involved in MSC differentiation toward the osteogenic lineage. ObI-1 encodes for a nuclear protein subjected to proteasomal degradation and expressed during osteoblast differentiation both in a murine multipotent mesenchymal cell line (W20-17) and in primary murine MSCs. RNA interference-mediated knockdown of ObI-1 expression significantly impairs osteoblast differentiation and matrix mineralization with reduced expression of the osteogenic markers, Runtrelated transcription factor 2 (Runx2) and osteopontin. Conversely, ObI-1 overexpression enhances osteogenic differentiation and bone-specific markers expression. ObI-1 stimulates bone morphogenetic protein (BMP)-4 expression and the consequent activation of the Smad pathway; treatment with a BMP receptor type I antagonist completely abolishes ObI-1-mediated stimulation of osteogenic differentiation. Collectively, our findings suggest that ObI-1 modulates osteogenic differentiation, at least in part, through the BMP signaling pathway, increasing Runx2 activation and leading to osteoblast commitment and maturation.

Keywords: osteogenic differentiation, KRAB, zinc-finger transcription factor, bone morphogenetic proteins, Runx2

\section{Introduction}

$\mathrm{O}$ STEOGENIC DIFFERENTIATION IS a sophisticated and tightly regulated biological process. In response to specific stimuli, mesenchymal stem cells (MSCs) differentiate into osteoblasts, the "bone-forming" cells, through a multistep process characterized by four major phases: commitment toward the osteogenic lineage, osteoprogenitor cells proliferation, osteoblast maturation, and bone matrix mineralization. Induction of the differentiation process and progression through the different steps are driven by a complex network of cytokines, hormones, and growth factors that modulates expression and activity of transcription factors and other bone-related proteins [1,2].

Among growth factors involved in osteogenic regulation, bone morphogenetic proteins (BMPs) play a role of paramount relevance in inducing bone and cartilage development $[3,4]$. BMPs belong to the transforming growth factor-beta superfamily and signal through two types of transmembrane serinethreonine kinase receptors, type I and type II [BMP receptor type I (BMPR-I) and BMP receptor type II BMPR-II], which, upon ligand binding, activate downstream signaling pathways such as Smad1/5/8 and MAPK pathways [5]. BMPs locally synthesized accumulate in the extracellular matrix and play a critical role in both inducing commitment of MSCs toward the osteogenic lineage and enhancing the activity of mature osteoblasts [6-8].

Bone morphogenetic proteins induce the expression of several genes, including transcription factors that control the genetic programs required for commitment and differentiation toward the osteoblastic pathway [5]. Among them, Runt-related transcription factor 2 (Runx2), although

${ }^{1}$ CEINGE-Biotecnologie Avanzate, Naples, Italy.

${ }^{2}$ Dipartimento di Medicina Molecolare e Biotecnologie Mediche, Università degli Studi di Napoli "Federico II," Naples, Italy.

${ }^{3}$ SEMM-European School for Molecular Medicine, Naples, Italy.

${ }^{4} \mathrm{CRG}$ - Centre for Genomic Regulation, Barcelona, Spain.

${ }^{5}$ Dipartimento di Sanità Pubblica, Università degli Studi di Napoli "Federico II," Naples, Italy.

*Current affiliation: Department of Cellular and Molecular Physiology, Institute of Translational Medicine, University of Liverpool, Liverpool, United Kingdom.

${ }^{\dagger}$ These authors contributed equally to this work. 
expressed also in other cell types, is considered as one of the most important transcriptional regulators of osteogenic differentiation. Indeed, Runx 2 is the earliest and most specific marker of osteogenesis, and its expression is absolutely required for osteoblast differentiation; Runx 2 ablation in mice results in a skeleton constituted mainly by cartilage with lack of bone due to the absence of osteoblasts [9-11].

In addition, Runx 2 haploinsufficiency in humans causes a condition known as cleidocranial dysplasia, characterized by dental anomalies and delayed skeletal development [11-13]. Runx2 is not only necessary for MSC commitment toward the osteogenic lineage but it also regulates osteoblast maturation and functions, such as matrix formation and mineralization, inducing expression of several osteoblastspecific genes, including alkaline phosphatase (ALP), type I collagen, osteopontin (OPN), and osteocalcin $[14,15]$. Therefore, due to its prominent role throughout osteogenic differentiation, Runx2 is regarded as the master gene of bone formation. BMPs induce Runx2 expression and stimulate its function, and both Smad-dependent and -independent signaling pathways activated by these factors converge to Runx 2 activation to modulate osteoblast differentiation $[16,17]$.

However, despite the efforts to elucidate the molecular mechanisms underlying osteogenic differentiation, our knowledge of this complex biological process is still incomplete. Indeed, a detailed understanding of the molecular processes that drive differentiation of MSCs toward the osteogenic lineage is essential to identify new molecular targets and develop novel and more effective therapeutic strategies for the treatment of bone-related pathologies, such as osteopenia/osteoporosis, bone fractures, and osteosarcoma.

Moreover, by virtue of their potential to differentiate into several mesenchymal lineages and their favorable immunemodulatory and anti-inflammatory properties, MSCs have emerged as a promising cell source for a wide range of regenerative medicine and tissue engineering applications and are largely used in preclinical and clinical studies to treat a broad spectrum of conditions [18]. Therefore, a thorough evaluation and comprehension of the molecular mechanisms regulating MSC self-renewal and differentiation toward a specific cell lineage is of paramount importance to optimize the use and fully exploit the potential of MSCs in clinical applications.

To identify genes involved in osteoblast differentiation, we have previously performed a high-throughput screening based on an RNA-interference (RNAi) approach. The murine bone marrow-derived stromal cell line W20-17 was used as cellular model and differentiated into osteoblasts, and mineral matrix deposition was chosen as read-out [19].

The present report focuses on the characterization of a previously undescribed transcription factor involved in osteogenic regulation, identified during this screening, that we named osteoblast inducer (ObI)-1. Subsequently to our screening, Jin et al. showed that the same gene is expressed in mouse gonads and the encoded protein is localized in the nucleus [20]. We also observed nuclear localization in primary murine MSCs (mMSCs) and showed that ObI-1 expression increases during osteogenic differentiation in W20-17 cells and in mMSCs, and its knockdown impairs this biological process. In addition, we observed that ObI-1 acts, at least in part, by stimulating BMP signaling and Runx2 expression. These findings identify a novel player of the BMPRunx2 axis regulating MSCs osteogenic differentiation.

\section{Materials and Methods}

\section{Cell cultures}

W20-17 cell line was obtained from American Type Culture Collection (Cat. No. CRL-2623/LGC standards; Milan, Italy). Primary mMSCs have been previously isolated from bone marrow of C57Bl/6 mice and characterized [21]. $\mathrm{Hu}-$ man MSCs (hMSCs) were isolated from bone marrow of healthy adult donors after informed consent according to the procedure established by the local Bioethics Institutional Committee [22]. mMSCs and W20-17 cells were seeded at a density of 6,000 cells $/ \mathrm{cm}^{2}$ in regular medium consisting of DMEM (COD. ECM0749L Euroclone, Siziano, Italy) supplemented with $10 \%$ fetal bovine serum (FBS; HyClone, Northumberland, United Kingdom) and $4 \mathrm{mM}$ L-glutamine (Gibco, Paisley, United Kingdom) in absence of antibiotics, and cultured for a limited number of passages $(\leq 10-15)$. hMSCs were seeded at a density of 5,000 cells $/ \mathrm{cm}^{2}$ and cultured in regular medium consisting of D-MEM supplemented with $20 \%$ FBS and $4 \mathrm{mM}$ L-glutamine in absence of antibiotics for a limited number of passages $(\leq 5)$. For osteoblastic differentiation, cells were grown until they reached $80 \%-90 \%$ confluence and then cultured in osteogenic medium consisting of D-MEM with $10 \%$ FBS, $4 \mathrm{mM}$ L-glutamine, $50 \mu \mathrm{M}$ ascorbic acid 2-phosphate, $10 \mathrm{mM}$ glycerol 2-phosphate, and $1 \mu \mathrm{M}$ dexamethasone (all from Sigma-Aldrich, St. Louis, MO) for the times indicated in the "Results" section.

For adipogenic differentiation of W20-17 cells, cells were grown until they reached $80 \%-90 \%$ confluence and then cultured in D-MEM with $10 \% \mathrm{FBS}, 4 \mathrm{mM}$ L-glutamine, $1 \mu \mathrm{M}$ dexamethasone, $100 \mu \mathrm{M}$ indometacin, $10 \mu \mathrm{g} / \mathrm{mL}$ insulin, and $0.5 \mathrm{mM}$ 3-isobutyl-1-methylxanthine (all from Sigma-Aldrich) for 10 days.

\section{Small hairpin RNA-mediated screening}

The high-throughput screening procedure used to identify novel genes potentially relevant for osteoblast differentiation has been previously described [19]. In brief, W20-17 cells were plated in 96-well plates and transfected with different small hairpin RNA (shRNA)-expressing plasmids (Open Biosystem, Huntsville, AL) using Lipofectamine 2000 (Invitrogen, Paisley, United Kingdom) according to manufacturer's instructions. Cells were then cultured in osteogenic differentiation medium for 21 days and mineral deposition was evaluated with alizarin red staining. Genes that, when silenced, impaired the ability of W20-17 cells to produce a mineralized matrix were considered potential candidate genes and further analyzed.

\section{Alizarin red and ALP staining}

For alizarin red staining, cells were washed with phosphate-buffered saline (PBS) and fixed in 10\% formaldehyde (Sigma-Aldrich) for $1 \mathrm{~h}$; after rinsing with distilled water, they were incubated with $2 \%$ alizarin red S (SigmaAldrich) at $\mathrm{pH} 4.1$ for $10 \mathrm{~min}$. Excess staining was removed 
using water. For W20-17 cell cultures, after visual examination the mineralized deposit-bound dye was extracted adding $100 \mu \mathrm{L}$ of $4 \mathrm{M}$ guanidine-hydrochloric acid (SigmaAldrich) and incubating them over night at room temperature. A semi-quantitative assay was used to determine the amount of cell-bound dye assessing absorbance at $490 \mathrm{~nm}$ of a 10-times dilution of the resulting supernatant. For ALP staining, cells were washed with PBS and fixed in $10 \%$ cold neutral formalin buffer ( $10 \%$ formalin, $0.1 \mathrm{M} \mathrm{Na}_{2} \mathrm{HPO}_{4}, 0.029 \mathrm{M}$ $\mathrm{NaH}_{2} \mathrm{PO}_{4}$ (Sigma-Aldrich). Cells were then rinsed with distilled water and stained with a substrate solution containing $0.24 \mathrm{mM}$ naphthol AS MX-PO 4 (Sigma-Aldrich), 0.4\% N,Ndimethylformamide (Sigma-Aldrich), and $1.6 \mu \mathrm{M}$ red violet LB salt (Sigma-Aldrich) in 0.2 M Tris-HCl pH 8.3 (Carlo Erba Reagenti, Milano, Italy) for $45 \mathrm{~min}$ at room temperature. Excess staining was removed washing twice with distilled water and ALP-positive cells visualized under microscope.

\section{shRNA transfection}

Murine MSCs or W20-17 cells were grown until they reached $90 \%$ confluence and then transfected with shRNAs from the same library used in the screening (Open Biosystem, Huntsville, AL) against ObI-1 gene or a scrambled shRNA [non-silencing shRNA (shNS)] as a control using Lipofectamine 2000, according to manufacturer's instructions. Two days after transfection, cells were plated in selective media containing $2 \mu \mathrm{g} / \mathrm{mL}$ puromycin (Sigma-Aldrich), and the medium was replaced every 3 days. A plate of untransfected cells was used as a control for the selection.

\section{Construction of Obl-1 Flag-expressing plasmid and stable transfection}

RNA was extracted from mouse lungs and retro-transcribed in cDNA as described below.

The following primers were used to amplify ObI-1 $(\approx 2$ $\mathrm{KB}$ ): Forward (BamHI restriction site-containing) 5'-GGCC ACGGGATCCAGCCATGGTGAGAAGACG-3' and Reverse (NotI restriction site-containing) 5'-GGGCTCGCGGCC GCCAAATCCCAGATAGGGTTTG-3'. We used for PCR amplification Phusion Taq polymerase (New England Biolabs, EuroClone, Pero, Italy) with the following protocol: $98^{\circ} \mathrm{C}$ for $3 \mathrm{~min}, 15$ cycles at $98^{\circ} \mathrm{C}$ for $1 \mathrm{~min}, 49^{\circ} \mathrm{C}$ for $20 \mathrm{~s}$, and $72^{\circ} \mathrm{C}$ for $2 \mathrm{~min}, 5$ cycles at $98^{\circ} \mathrm{C}$ for $1 \mathrm{~min}, 60^{\circ} \mathrm{C}$ for $20 \mathrm{~s}$, and $72^{\circ} \mathrm{C}$ for $2 \mathrm{~min}, 15$ cycles at $98^{\circ} \mathrm{C}$ for $1 \mathrm{~min}$ and $72^{\circ} \mathrm{C}$ for $2 \mathrm{~min}$, and a final extension at $72^{\circ} \mathrm{C}$ for $10 \mathrm{~min}$.

The amplified product was resolved by agarose gel electrophoresis (1\% agarose with $0.5 \mu \mathrm{g} / \mathrm{mL}$ ethidium bromide; Sigma-Aldrich); the band was purified using Wizard SV Gel and PCR Cleanup system (Promega Italia Srl, Milano, Italy). PCR product and pcDNA3.1 vector (Invitrogen) were digested for $2 \mathrm{~h}$ at $37^{\circ} \mathrm{C}$ with $B a m \mathrm{HI}$ and NotI (New England Biolabs, EuroClone), resolved by agarose gel electrophoresis and extracted.

Vector dephosphorilation and ligation reactions were performed using the Rapid Dephosphorilation and Ligation kit (Roche, Milano, Italy) according to manufacturer's instructions. The ligation mixture was transformed into calcium chloride competent DH5 $\alpha$ cells (Invitrogen). Plasmids that after BamHI-NotI digestion showed that the presence of a $2 \mathrm{~KB}$ insert were validated by sequencing (Ceinge Sequencing Service). mMSCs were grown until they reached
$90 \%$ confluence and then transfected with either ObI-1 Flagexpressing plasmid or pcDNA3.1 empty vector using Lipofectamine 2000 and, after 2 days, plated in selective media containing $400 \mu \mathrm{g} / \mathrm{mL} \mathrm{G418} \mathrm{(Gibco);} \mathrm{the} \mathrm{medium}$ was replaced every 3 days. A plate of untransfected cells was used as a control for the selection.

\section{Mice experiments}

C57B1/6 mice were obtained from Jackson Laboratory (Bar Harbor, ME). Experiments were performed in conformity with protocols approved by the veterinary department of the Italian Ministry of Health and in accordance with the ethical and safety rules and guidelines for the use of animals in biomedical research provided by the relevant Italian laws and European Union's directives (no. 86/609/EC). All efforts were made to minimize the animals' suffering. Mice were euthanized with carbon dioxide vapors; organs and tissues were immediately collected in ice and homogenized in Tri Reagent (Sigma-Aldrich).

\section{RNA extraction and real-time PCR}

Total RNA was extracted from cells or murine tissues and organs using Tri Reagent (Sigma-Aldrich) according to manufacturer's instructions. cDNA was amplified from $2 \mu \mathrm{g}$ of RNA using M-MuLV reverse transcriptase (New England Biolabs, EuroClone). The following protocol was performed: RNA was incubated with dNTPs (Amersham Biosciences) and Random Examers (Promega Italia Srl) at $70^{\circ} \mathrm{C}$ for $10 \mathrm{~min}$ and in ice for $1 \mathrm{~min}$; then, after the addition of $\mathrm{M}-\mathrm{MuLV}$ and the specific buffer, the incubation was performed at $42^{\circ} \mathrm{C}$ for $1 \mathrm{~h}$ and at $90^{\circ} \mathrm{C}$ for $10 \mathrm{~min}$. Real-time PCR was performed using the SYBR Green PCR master mixture in an ABI PRISM 7500 apparatus (Applera, Foster City, CA). Levels of target genes were quantified using specific oligonucleotide primers and normalized for glyceraldehyde-3-phosphate dehydrogenase (GAPDH) or ribosomal protein L13A (RPL13A) expression. Primer sequences are available upon request.

\section{Immunofluorescence analysis}

Murine MSCs were grown until they reached 60\%-70\% confluence and then transfected with either pcDNA3.1 expressing ObI-1 Flag or an empty vector using Lipofectamine 2000. After $24 \mathrm{~h}$, cells were fixed in $4 \%$ paraformaldehyde (Sigma-Aldrich), permeabilized with $0.2 \%$ Triton X-100 (Sigma-Aldrich) and blocked in $10 \%$ bovine serum albumin (BSA) in PBS for $30 \mathrm{~min}$ at room temperature. The samples were incubated with primary anti-Flag antibody (mouse monoclonal, Cat. No. F1804, 1:250 dilution; Sigma-Aldrich) and then with an anti-mouse immunoglobulin $\mathrm{G}$ (IgG) conjugated with Alexa Fluor 488 (Molecular Probes, Eugene, OR; Cat. No. A-21202, 1:500 dilution). Both antibodies were diluted in $3 \% \mathrm{BSA} / \mathrm{PBS}$. Cells nuclei were counterstained with 4',6-diamidino-2-phenylindole (DAPI) diluted 1:5,000 in PBS. Images were captured using a confocal microscope (LSM 510 Meta; Zeiss).

\section{Bromo-2'-deoxyuridine incorporation assay}

Murine MSCs were plated at 50\% confluence and the following day transfected with either stealth small interfering RNAs (siRNAs) against ObI-1 or scramble negative control 
siRNA (20 nM, NS\# 462001, 1\# 291188A08, 2\# 291188A10; Invitrogen) using Lipofectamine 2000 (Invitrogen) according to manufacturer's instructions. Twenty-four hours after transfection, cells were cultured in regular media or complete osteogenic medium for the next $24 \mathrm{~h}$. Cell proliferation was then assessed performing a bromo- $2^{\prime}$-deoxyuridine (BrdU) incorporation assay (BrdU labeling and detection kit I; Roche) according to manufacturer's instructions; cells were incubated in the BrdU labeling medium for $40 \mathrm{~min}$ at $37^{\circ} \mathrm{C}$. Cells nuclei were counterstained with DAPI diluted 1:5,000 in PBS and the percentage of BrdU-positive nuclei was determined by counting 30 fields $(10 \times$ magnification).

\section{Proteasomal inhibition}

Murine MSCs, stably transfected with either pcDNA3.1 empty vector or the ObI-1 Flag-expressing plasmid, were seeded at a density of $19,000 \mathrm{cells} / \mathrm{cm}^{2}$ and the following day treated with either dimethyl sulfoxide (DMSO, control) or proteasome inhibitor MG 132 (Sigma-Aldrich) at 5 or $10 \mu \mathrm{M}$ concentration. After $6 \mathrm{~h}$, cells were lysed and protein collected for further analyses.

\section{Cycloheximide chase assay}

Murine MSCs were grown until they reached $70 \%-80 \%$ confluence and then transfected with either pcDNA3.1 expressing ObI-1 Flag or the empty vector using Lipofectamine 2000. After $24 \mathrm{~h}$, cells were treated with either DMSO (control) or $10 \mu \mathrm{g} / \mathrm{mL}$ cycloheximide (Sigma-Aldrich) and proteins collected at different time points and subjected to western blot analysis.

\section{BMP-4 pathway stimulation}

Murine MSCs, stably transfected with either pcDNA3.1 empty vector or ObI-1 Flag-encoding plasmid, were seeded at a density of 19,000 cells $/ \mathrm{cm}^{2}$ and starved overnight in DMEM supplemented with 2\% FBS to reduce cell proliferation. The following day, cells were treated with $40 \mathrm{ng} / \mathrm{mL}$ recombinant BMP-4 (Sigma-Aldrich) and proteins collected at different time points and subjected to western blot analysis. Unstimulated cells were used as control. Optimization experiments have been performed to select the BMP-4 concentration able to induce a robust and reproducible activation of the Smad pathway (Smad1/5/8 phosphorylation) in our cell system (data not shown).

\section{BMPR-I inhibition}

Murine MSCs, stably transfected with either pcDNA3.1 empty vector or, ObI-1 Flag-encoding plasmid, were grown until they reached $80 \%-90 \%$ confluence and then were cultured in regular medium, osteogenic medium alone, or supplemented with the BMPR-I antagonist LDN193189 (Sigma-Aldrich) at a concentration of $100 \mathrm{nM}$, chosen on the basis of previous reports [23,24]. LDN193189 was added to the culture medium every $48 \mathrm{~h}$ during osteogenic differentiation. After 14 days of differentiation, alizarin red staining was performed to evaluate mineral matrix deposition.

\section{Western blot assays}

Total cell lysates were obtained by treatment with lysis buffer ( $1 \mathrm{mM}$ ethylenediaminetetraaceticacid, $50 \mathrm{mM}$ Tris-
$\mathrm{HCl}, \mathrm{pH} 7.5,70 \mathrm{mM} \mathrm{NaCl}, 1 \%$ Triton X-100), protease inhibitors (Complete Protease Inhibitor Cocktail; Roche), and phosphatase inhibitors sodium fluoride $(50 \mathrm{mM})$ and sodium orthovanadate (1 mM; Sigma-Aldrich). Proteins were quantified with the Bradford solution according to manufacturer's instructions (AppliChem, Darmstadt, Germany).

Protein extracts were electrophoresed on $10 \%$ sodium dodecyl sulfate-polyacrylamide gel electrophoresis gels, transferred on Immuno-Blot polyvinylidene fluoride membranes (Bio-Rad, Hercules, CA) and analyzed using the following antibodies: anti-Flag (mouse monoclonal, Cat. No. F1804, 1:1,000 dilution; Sigma-Aldrich), anti-GAPDH (mouse monoclonal, Cat. No. sc-32233, 1:1,000 dilution; Santa Cruz Biotechnology), anti-Ubiquitin (Ub, rabbit polyclonal, Cat. No. sc-9133, 1:700 dilution; Santa Cruz Biotechnology), anti-p53 (rabbit polyclonal, Cat. No. sc6243, 1:1,000 dilution; Santa Cruz Biotechnology), antipSmad1/5/8 (rabbit monoclonal, 1:1,000 dilution, Cat. No. 9516; Cell Signaling, Danvers, MA), anti-Smad1 (rabbit polyclonal, Cat. No. 9743, 1:1,000 dilution; Cell Signaling), anti-HA (mouse monoclonal, Cat. No. sc-7392, 1:1,000 dilution; Santa Cruz Biotechnology), and secondary horseradish peroxidase-conjugated antibody (Amsterdam Bioscience, Uppsala, Sweden; 1:5,000 dilution). Proteins were detected by chemiluminescence (ECL or ECL plus; GE Healthcare, United Kingdom). Quantitative analysis was performed using ChemiDoc (Bio-Rad) according to manufacturer's instructions. pSmad1/5/8 levels were normalized to Smad1 levels and results are expressed as fold change of normalized pSmad1/5/8 levels in BMP4-treated cells at different time points versus untreated cells.

\section{Immunoprecipitation}

Murine MSCs, stably transfected with the ObI-1 Flag expressing vector or pcDNA3.1 empty vector, were grown until they reached $90 \%$ confluence and then transiently transfected with HA-Ub-encoding pcDNA3 plasmid (HAUb). HA-Ub (Addgene plasmid No. 18712) was a gift from Yeh [25]. Twenty-four hours after transfection, cells were treated with a $5 \mu \mathrm{M}$ concentration of MG 132. Six hours after treatment, cell extracts were collected and subjected to western blot analysis. HA-Ub-untransfected mMSCs and mMSCs treated with either DMSO or MG 132 were included as controls.

Protein lysate $(1.5 \mathrm{mg})$ was subjected to immunoprecipitation (IP). A preclearing step with an irrelevant antibody (mouse IgG1 isotype control, Cat. No. ab81032; Abcam) was performed to reduce nonspecific binding. Cell lysates were then incubated with $3.75 \mu \mathrm{g}$ of anti-Flag antibody (Sigma-Aldrich) for $3 \mathrm{~h}$ at $4^{\circ} \mathrm{C}$ and with protein $\mathrm{A} / \mathrm{G}-$ agarose (Santa Cruz Biotechnology; Cat. No. sc-2003) overnight at $4^{\circ} \mathrm{C}$. The following day, samples were washed three times and analyzed by western blot as previously described.

\section{Statistical analysis}

Data are reported as mean \pm standard deviation of three independent experiments. Two-tailed Student's $t$-test was performed using GraphPad Prism 5.0 software (San Diego, CA). A $P$-value $<0.05$ was considered statistically significant. 


\section{Results}

\section{Obl-1 is a putative transcription factor with increasing expression during osteoblast differentiation}

To identify genes that regulate osteoblast differentiation, we have previously used single clones from a library of shRNA-expressing plasmids able to silence specific murine genes in the multipotent murine cell line W20-17, as previously published [19]. We also used the same library to successfully identify genes involved in other biological processes, such as murine embryonic stem cell self-renewal or differentiation [26,27]. With this approach, we identified 650 candidate genes that, when silenced, impair the ability of W20-17 cells to differentiate in osteoblasts and produce mineralized matrix [19].

Among the identified genes, we focused on the characterization of a previously undescribed gene (that we named ObI-1), and its role in osteogenic differentiation. ObI-1 (A430033k04Rik) belongs to the Riken family of transcripts and is predicted to encode for two short and two long isoforms originating from alternative splicing (Supplementary Fig. S1A). The shortest isoforms are predicted to be $\sim 100$ aa in length (Supplementary Fig. S1B, C) and contain a Kruppel-associated box (KRAB) domain usually associated to transcriptional repression [28]. The long isoforms contain, in addition to the amino-terminal KRAB domain, several zinc-finger domains at the C-terminus
(Supplementary Fig. S1D, E). The presence of both an effector and a DNA-binding domain suggests that ObI-1 long isoforms could play a role as transcriptional regulators.

According to the expressed sequence tag (EST) profiles on UniGene database of NCBI, ObI-1-matching ESTs are present in several murine adult tissues, including bone marrow, as well as during embryonic development (Supplementary Fig. S2).

Subsequently, we investigated ObI-1 expression during osteogenic differentiation in vitro. Real-time PCR analysis in W20-17 cells indicates that the ObI-1 long isoform (A430033K04Rik-002) transcript levels increase from the first days of differentiation and remain high during the whole process. On the contrary, both the short forms and the other long isoform (A430033K04Rik-001) were almost undetectable, indicating that they may represent only a bioinformatic prediction or they are tissue-specific isoforms not expressed in MSCs nor involved in osteogenic differentiation (Fig. 1A and data not shown). Therefore, these isoforms were not further investigated.

ObI-1 long form (A430033K04Rik-002) expression profile during osteogenic differentiation of primary mMSCs, isolated from bone marrow, is comparable to what observed in W20-17, with transcript levels significantly increasing during the first days of differentiation (Fig. 1B). This finding further supports a role for ObI-1 gene in osteogenic differentiation. Moreover, ObI-1 transcription appears to be selectively up-regulated during osteogenic differentiation
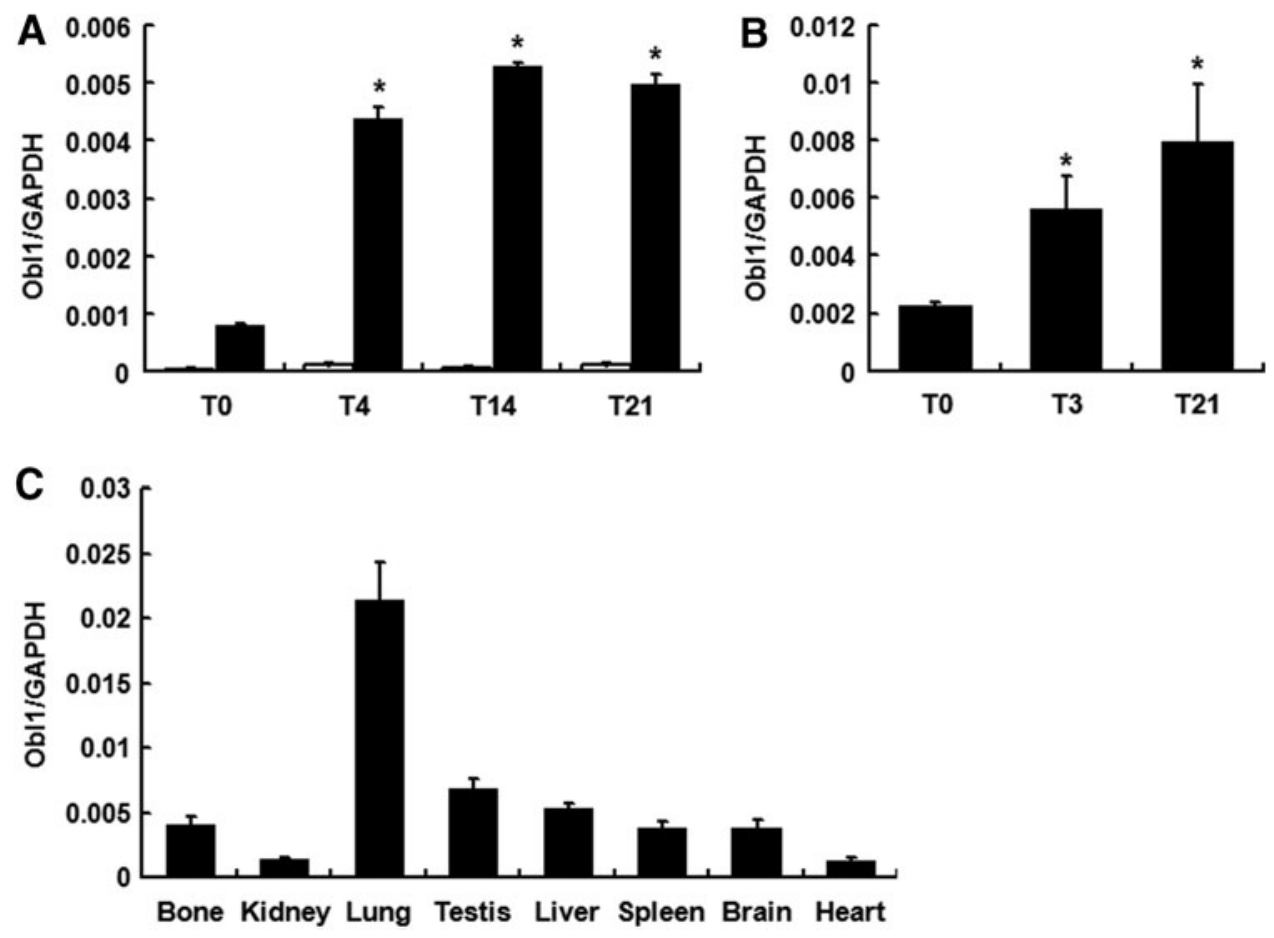

FIG. 1. ObI-1 is expressed during osteogenic differentiation and in several murine tissues. Expression profiles of ObI-1 transcripts during osteogenic differentiation of (A) W20-17 cells at day 0, 4, 14, and 21 and of (B) primary bone marrow-derived mMSCs at day 0,3, and 21. (A) ObI-1 long isoform levels (black bars) significantly increase in differentiating W2017 cells from day 4, whereas ObI-1 short-form levels (white bars) are barely detectable. (B) ObI-1 long transcript levels significantly increase also during osteogenic differentiation of mMSCs. (C) Real-time PCR analysis shows that ObI-1 is expressed in several mouse tissues and organs, with highest levels in lung. Results are expressed as ObI-1/GAPDH levels ratio $(* P<0.05)$. mMSC, murine mesenchymal stem cell; GAPDH, glyceraldehyde-3-phosphate dehydrogenase; ObI, osteoblast inducer. 
of MSCs compared to other differentiation fates, as its transcript levels decrease during adipogenic differentiation of W20-17 cells (Supplementary Fig. S3).

Recently, studies have shown that some of the most popular housekeeping genes, used as internal controls to normalize the expression of genes of interest in real time (RT)-quantitative polymerase chain reaction experiments, do not have a stable expression during MSC differentiation, although it is still unclear which housekeeping gene is the most appropriate and reliable reference gene [29,30]. Therefore, we decided to analyze ObI-1 expression profile during osteogenic differentiation of mMSCs using as a reference gene the ribosomal protein RPL13A in addition to GAPDH. We chose RPL13A among other housekeeping genes since it has been previously shown to have a stable expression in bone marrow tissues and during differentiation of MSCs toward the osteogenic lineage [30,31]. Our results confirmed that ObI-1 transcript levels increase during osteogenic differentiation, regardless of the reference gene used for the normalization (Supplementary Fig. S4A). Indeed, the expression levels of ObI-1 as well as the osteogenic markers Runx2 and OPN normalized to RPL13A or GAPDH were very similar (Supplementary Fig. S4B, C).

Moreover, we observed that ObI-1 expression is not restricted to osteoblasts; Real-time PCR analysis in several murine tissues showed a broad expression pattern since ObI-1 transcript could be detected at variable levels in all the tissues examined. Sur- prisingly, we observed the highest levels of expression in lung (Fig. 1C). This finding suggests that ObI-1 could play a role in different biological processes in a number of tissues.

We performed a phylogenetic analysis of the murine ObI-1 gene to identify potential orthologous genes in other species using MetaPhOrs, a public repository of phylogeny-based orthology and paralogy predictions computed using resources available in seven different homology prediction services [32]. Potential ObI-1 orthologs were identified in several species, including Human (Homo sapiens), Macaque (Macaca mulatta), Orangutan (Pongo abelii), and Rat (Rattus Norvegicus). In particular, the predicted human ortholog corresponds to the zincfinger protein-717 (ZNF717) gene; this gene, similarly to ObI-1, contains KRAB and zinc-finger domains. Interestingly, ZNF717 expression also increases during osteoblast differentiation of hMSCs from day 3 (Supplementary Fig. S5); this observation supports the predicted orthology and, therefore, suggests a possible role of ZNF717 in human osteoblastogenesis.

\section{Obl-1 is a nuclear protein subjected to proteasomal degradation}

As previously described by Jin et al. [20], we confirmed ObI-1 nuclear subcellular localization in mMSCs: immunofluorescence analysis in mMSCs transiently transfected with a vector expressing ObI-1 fused to a Flag tag (ObI-1 Flag)
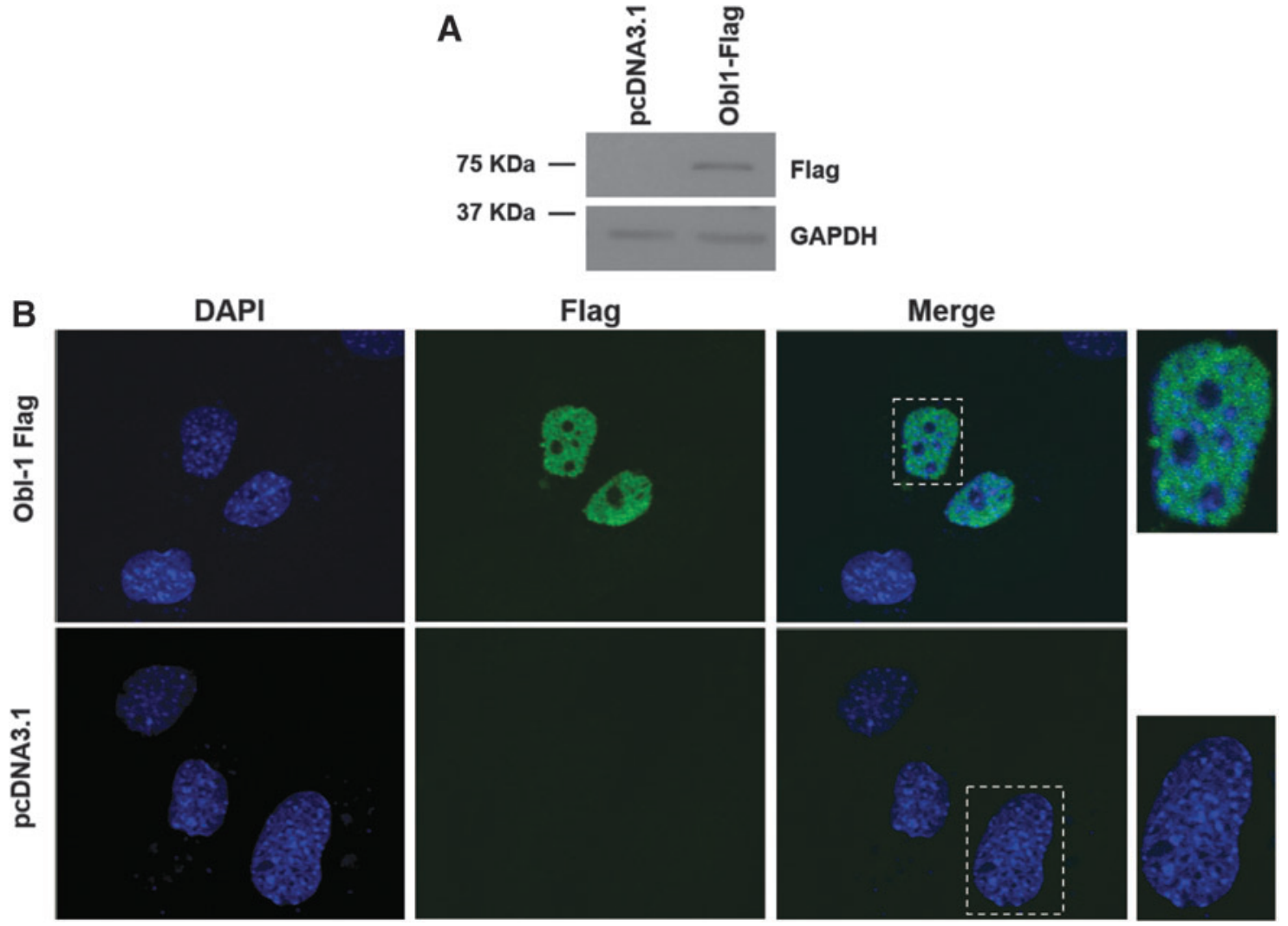

FIG. 2. ObI-1 is localized in the nucleus. (A) Western blot analysis confirms ObI-1 expression in mMSCs transfected with the pcDNA3.1 ObI-1 Flag-expressing plasmid $24 \mathrm{~h}$ after transfection. Cells transfected with the empty vector (pcDNA3.1) were used as negative control. GAPDH was used as control for protein loading. (B) ObI-1 subcellular localization was investigated in mMSCs transfected with either the ObI-1 Flag-expressing plasmid or the empty vector by immunofluorescence analysis with an anti-Flag antibody $24 \mathrm{~h}$ after cell transfection. Merging between the nuclear dye DAPI and the anti-Flag antibody indicates that the protein is localized in the cell nucleus. Magnification $63 \times$. Cells on the right represent a higher magnification of the cells indicated with a dotted line. DAPI, 4',6-diamidino-2-phenylindole. 

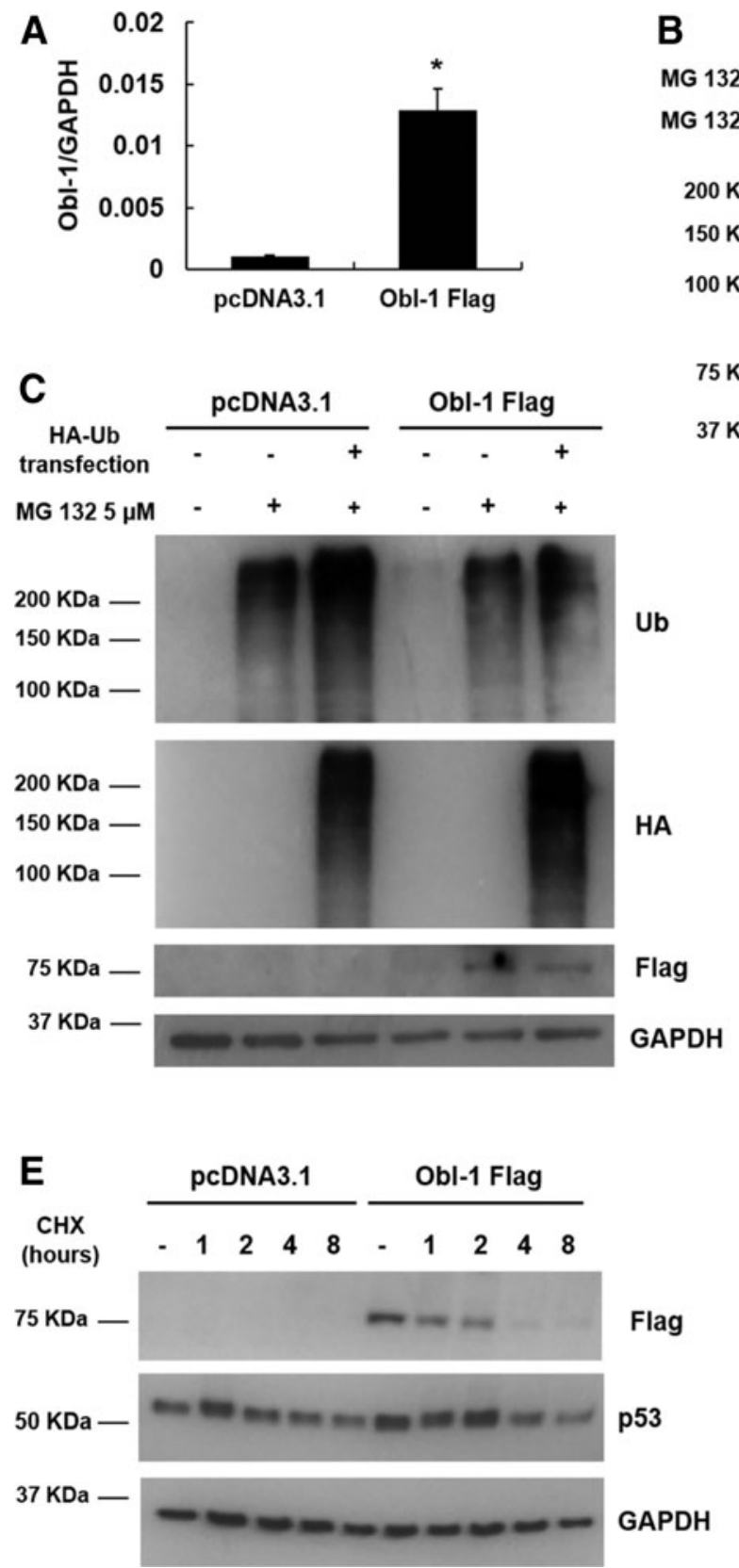
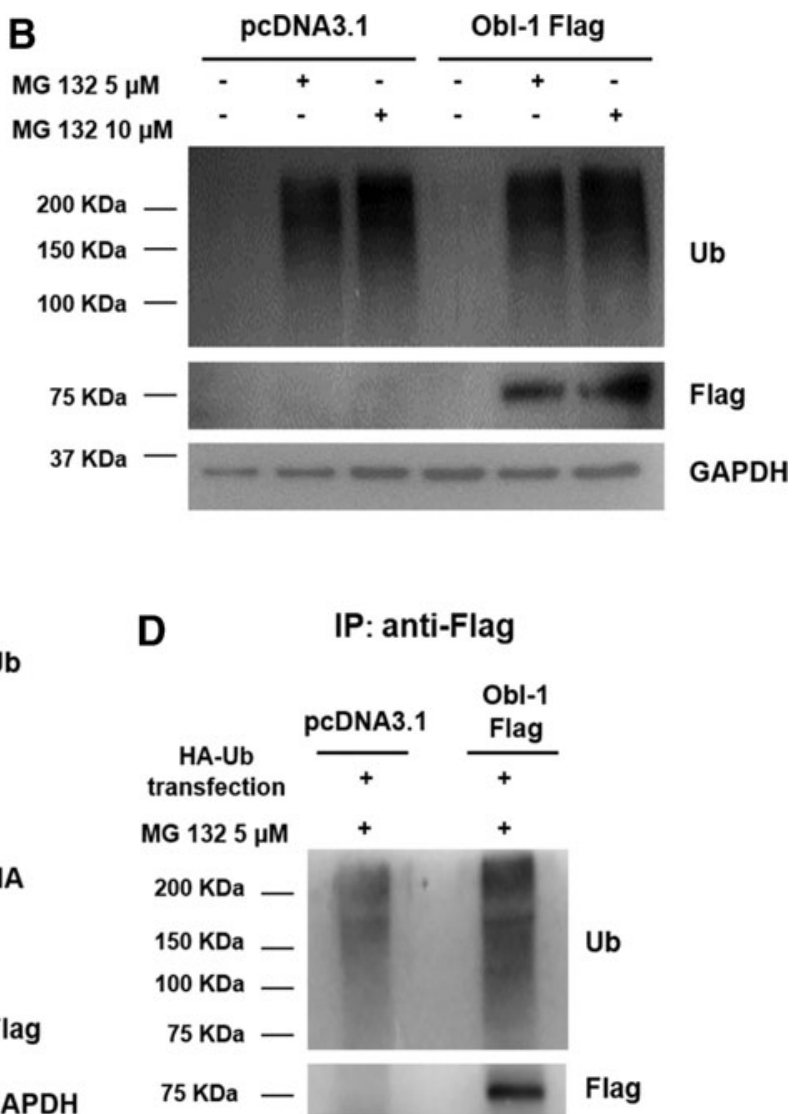

FIG. 3. ObI-1 protein is subjected to proteasomal degradation. (A) Real-time PCR analysis shows a significant increase in ObI-1 transcript levels in mMSCs stably transfected with ObI-1 Flag compared with cells transfected with the empty vector (pcDNA3.1). Results are expressed as ObI-1/GAPDH levels ratio $(* P<0.01)$. (B) Western blot analysis of cells transfected with either ObI-1 Flag or empty vector. Cells were treated with DMSO (control) or the proteasome inhibitor MG 132 (5 or $10 \mu \mathrm{M}$ ) for $6 \mathrm{~h}$. Whole cell extracts were probed with anti-Flag, anti-Ub, and anti-GAPDH antibodies. GAPDH levels were included as loading control. Proteasomal inhibition allows ObI-1 Flag protein accumulation, indicating that the protein is subject to proteasomal degradation. The accumulation of high molecular weight proteins reactive to anti-Ub indicates that MG 132 treatment was effective in reducing the degradation of Ub-conjugated proteins. (C) Cells stably transfected with either ObI-1 Flag or empty vector were transiently transfected with a vector expressing HA-Ub and treated with DMSO or $5 \mu \mathrm{M}$ MG132. Efficiency of HA-Ub transfection and MG 132 proteasomal inhibition were confirmed by western blot analysis on whole-cell extracts probed with anti-Ub, anti-HA, anti-Flag, and anti-GAPDH antibodies. (D) The same protein extracts were immunoprecipitated with anti-Flag antibody and probed with anti-Flag and anti-Ub antibodies. ObI-1 protein, accumulated following MG 132 treatment, is ubiquitylated. (E) Western blot analysis of mMSCs transiently transfected with either ObI-1 Flag or empty vector. Twenty-four hours after transfection, cells were treated with DMSO (control) or the translation inhibitor CHX $(10 \mu \mathrm{g} / \mathrm{mL})$ for $1,2,4$, or $8 \mathrm{~h}$. Whole-cell extracts were probed with anti-Flag, anti-p53, and antiGAPDH antibodies. GAPDH was used as loading control; p53 was included as a known unstable protein control. ObI-1 Flag protein levels rapidly decrease after CHX-induced inhibition of protein synthesis. DMSO, dimethyl sulfoxide; Ub, ubiquitin; $\mathrm{CHX}$, cycloheximide. 
showed that the protein is exclusively localized into the nucleus, thereby supporting a possible role in transcriptional regulation (Fig. 2).

We then attempted to generate mMSCs stably expressing the ObI-1 Flag protein. However, despite a robust expression of the ObI-1 transcript (Fig. 3A), we were not able to detect the corresponding protein through western blot analysis (data not shown and Fig. 3B). This result suggested that ObI-1, as most transcriptional factors, could be subjected to posttranslational modifications that regulate its stability and/or function [33].

To assess whether mechanisms such as Ub-mediated proteasomal degradation may be involved, we evaluated the presence of the ObI-1 Flag protein after inhibition of proteasomal function. Consistently with our hypothesis, treatment with the proteasome inhibitor MG 132 results in ObI-1 Flag protein accumulation in mMSCs (Fig. 3B). We then performed an IP analysis in mMSCs stably expressing ObI-1 Flag and transiently transfected with a vector expressing $\mathrm{HA}-\mathrm{Ub}$ to increase the cellular pool of free Ub. Efficiency of both HA-Ub transfection and MG 132 proteasomal inhibition were confirmed by western blot analysis (Fig. 3C). The same cell lysates were subjected to IP with an anti-Flag antibody, and western blot analysis confirmed that the ObI-1 protein, stabilized following MG 132 treatment, is polyubiquitylated (Fig. 3D).

These results indicate that ObI-1 protein levels, and therefore its function, are finely regulated by Ub-mediated proteasomal degradation, a mechanism commonly used by eukaryotic cells to tightly control the activity of transcription factors and other chromatin-associated proteins [34].

To further confirm this finding, we then performed a cycloheximide chase experiment in mMSCs transiently transfected with ObI-1 Flag or the empty vector to evaluate ObI-1 stability when translation, and therefore de novo protein synthesis, is inhibited. Consistently, we showed that ObI-1 encodes for a highly unstable, rapidly degraded protein (Fig. 3E).

\section{Obl-1 is necessary for osteoblast differentiation of $\mathrm{mMSCS}$}

Following ObI-1 identification, we confirmed the relevance of this gene for proper osteoblast maturation with additional silencing experiments, to ensure the specificity of the results and minimize the possibility of RNAi off-target effects. Therefore, we stably transfected W20-17 cells with three independent shRNAs that recognize different region of the ObI1 transcript, and observed an almost complete absence of matrix mineralization, as assessed by alizarin red staining after 21 days of osteogenic differentiation (Fig. 4A-E). Staining quantification showed a significant reduction of mineralized deposits in ObI-1 silenced cells compared with untransfected cells and W20-17 cells transfected with shNS, used as controls (Fig. 4F). Effective reduction of ObI-1 levels was confirmed by RT-polymerase chain reaction (RT-PCR) (Fig. 4G).

We evaluated ObI-1 involvement in osteogenic regulation also in primary mMSCs. As observed in W20-17 cells, ObI1 silencing significantly impairs the ability of cells to produce mineralized nodules 18 days after osteogenic induction (Fig. 4L-O and Supplementary Fig. S6). RT-PCR confirmed silencing efficiency (Fig. 4P).

To characterize the role of ObI-1 in osteoblast differentiation, we decided to investigate the phase of the dif- ferentiation process, in which this gene is required. We observed that ObI-1 silencing results in a reduced expression of ALP, a protein expressed by mature osteoblasts that promotes extracellular matrix mineralization and considered an intermediate marker of osteogenic differentiation, after 12 days of differentiation (Fig. 4H-K) [35].

This finding indicates that ObI-1 is not involved only in the mineralization phase, but its function is required in earlier steps of osteogenic differentiation. Furthermore, this result is also in agreement with the observation that ObI-1 is expressed early during osteogenic differentiation of both W20-17 and mMSCs (Fig. 1A, B).

The hypothesis that ObI- 1 could play a role during an early step of osteoblastogenesis is further corroborated by the observation that its silencing results in reduced levels of Runx2 and OPN during W20-17 cell differentiation (Fig. 5A, B). Indeed, the transcription factor Runx 2 is an early marker of osteogenesis, and it is absolutely required for the commitment of MSCs toward the osteogenic lineage as well as for the function of mature osteoblasts, whereas OPN is a bone matrix sialoprotein expressed throughout osteogenic differentiation, from the first day of the process, reaching a peak during the mineralization phase $[9,10,36]$. As the transcript levels of both markers were significantly reduced in ObI-1 silenced cells, ObI-1 is likely to be involved in an early phase of osteogenic commitment, acting probably upstream of Runx 2 .

We then evaluated the effects of ObI-1 silencing on osteoprogenitors proliferation, which represents the first step of osteogenic differentiation. Interestingly, ObI-1 silencing selectively impaired the proliferation of preosteoblasts, with a decreased BrdU incorporation $24 \mathrm{~h}$ after osteogenic induction compared with control cells, whereas the proliferation of undifferentiated MSCs was not affected (Fig. 6A, B). Silencing efficacy was confirmed by real-time PCR analysis $48 \mathrm{~h}$ after siRNA transfection (Fig. 6C). These findings suggest that ObI-1 specifically stimulates the proliferation of cells committed toward the osteogenic lineage, rather than being a general regulator of cell proliferation.

\section{Obl-1 expression stimulates osteoblast differentiation through a BMP-mediated mechanism}

We then evaluated whether overexpression of ObI-1 could enhance osteoblast differentiation in mMSCs. At this aim, we induced the osteogenic differentiation in mMSCs stably transfected with the ObI-1 Flag encoding plasmid and observed an increased mineralized matrix deposition compared with control cells (Fig. 7A-F and Supplementary Fig. S7A). Similar results were obtained when cells were cultured in medium supplemented only with ascorbic acid 2-phosphate and glycerol 2-phosphate without the osteogenic inducer dexamethasone, further indicating that ObI-1 expression enhances mMSCs commitment and/or differentiation toward the osteogenic lineage (Fig. 7B, E, and Supplementary Fig. S7A). In agreement with this finding, ObI-1 Flag transfected mMSCs showed increased levels of Runx2 and OPN compared with controls (Supplementary Fig. S8). Therefore, it is conceivable that ObI-1 favors osteoblastogenesis by directly or indirectly stimulating Runx 2 expression.

BMPs are among the most well-studied factors known to induce osteoblasts and chondrocytes differentiation also through the increase of Runx2 levels $[17,37,38]$. To investigate 

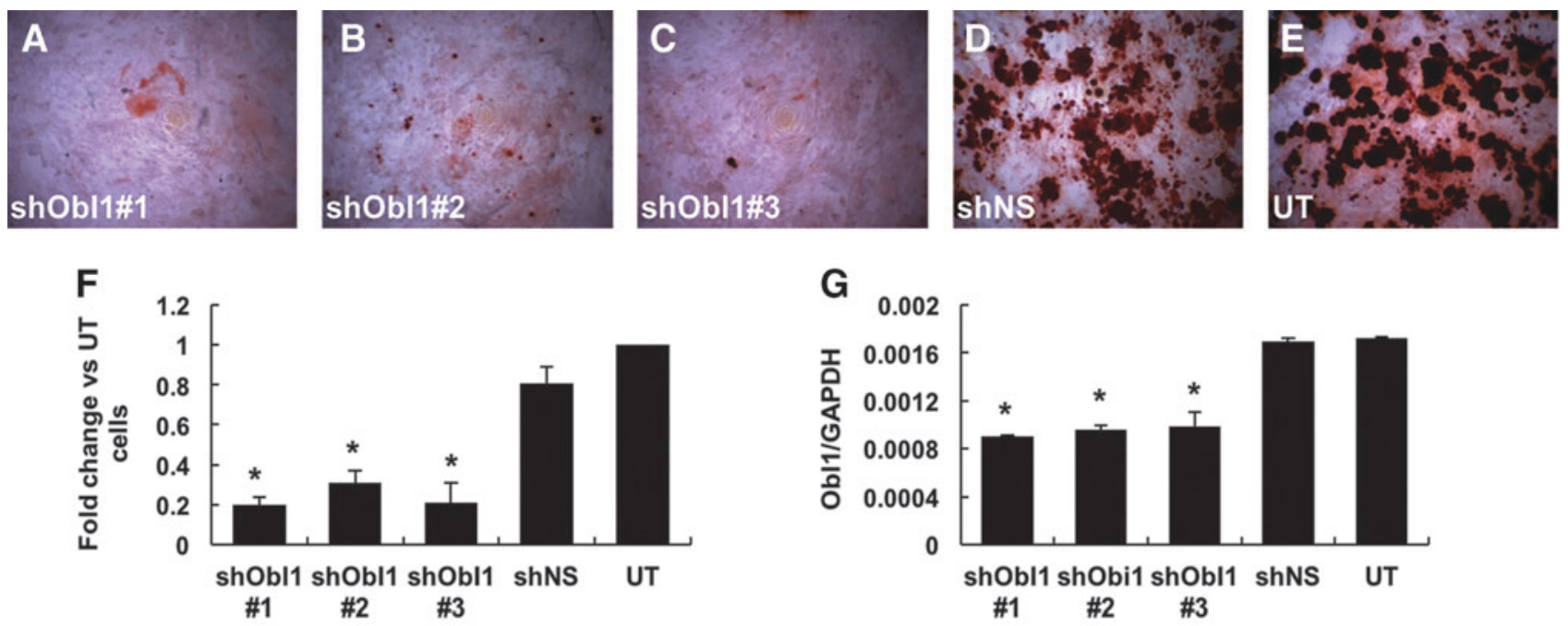

shNS
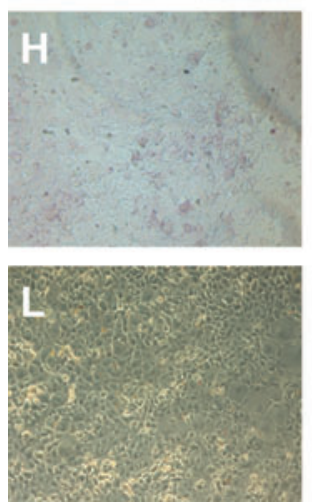
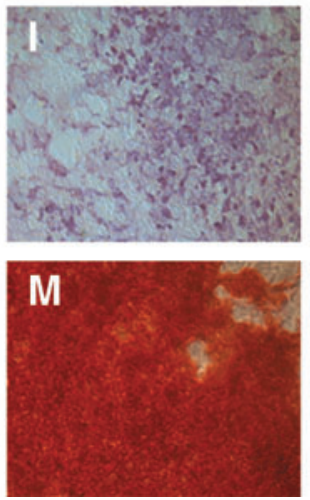

shObl1\#1
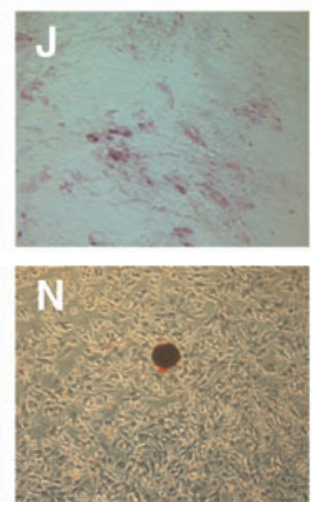

shObl1\#2

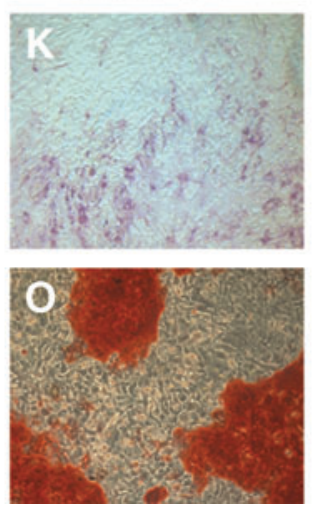

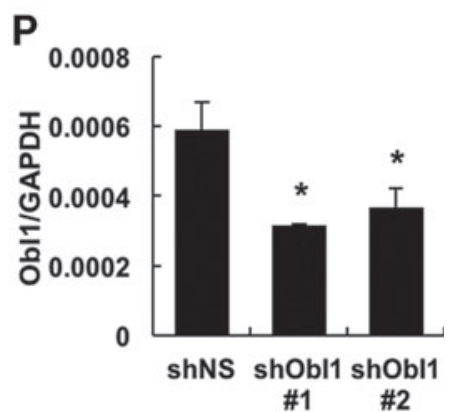

FIG. 4. ObI-1 gene silencing impairs osteoblast differentiation. W20-17 cells transfected with three different shRNAs against ObI-1 transcript, (A) shObI1\#1, (B) shObI1\#2, and (C) shObI1\#3, show a reduction of alizarin red staining compared with (D) cells transfected with shNS and (E) untransfected (UT) cells 21 days after differentiation induction (magnification $10 \times$ ). (F) Alizarin red dye quantification confirms microscopy data that mineral deposition is significantly impaired in ObI-1-silenced cells. (G) Effective silencing has been confirmed by real-time PCR analysis that shows a significant decrease in ObI-1 transcript levels in W20-17 cells transfected with shRNAs against ObI-1 compared with control cells. Results are expressed as ObI-1/GAPDH levels ratio. ObI-1 silencing impairs osteogenic differentiation of mMSCs: cells transfected with $(\mathbf{J}, \mathbf{N})$ shObI1\#1 and $(\mathbf{K}, \mathbf{O})$ shObI1\#2 and cultured with osteogenic medium show a reduction of ALP staining $(\mathbf{J}, \mathbf{K})$ after 12 days as well as a decrease of mineral deposition $(\mathbf{N}, \mathbf{O})$ assessed by alizarin red staining after 18 days of differentiation. mMSCs transfected with shNS and cultured with regular $(\mathbf{H}, \mathbf{L})$ and osteogenic (I, M) medium were used as controls. Magnification 10×. Pictures are representative of three independent experiments. (P) ObI1 silencing efficacy was confirmed by real-time PCR analysis: ObI-1 transcript levels are significantly reduced in mMSCs transfected with shObI1\#1 or shObI1\#2 compared with cells transfected with shNS. Results are expressed as ObI-1/ GAPDH levels ratio. $* P<0.05$. shRNA, small hairpin RNA; shNS, non-silencing shRNA; ALP, alkaline phosphatase.

whether ObI-1 affects the BMP pathway, we evaluated BMP-4 expression by RT-PCR and found that its transcript levels were significantly upregulated in mMSCs overexpressing ObI-1 compared with control cells (Fig. 7G). Conversely, ObI-1 silencing induced a decrease in BMP-4 levels (Supplementary Fig. S9).

We then evaluated whether ObI-1 expression influences BMPs signaling and Smad1/5/8 phosphorylation and, therefore, activation. mMSCs, stably transfected with either the ObI-1 Flag expression plasmid or a pcDNA3.1 empty vector, were treated with $40 \mathrm{ng} / \mathrm{mL}$ BMP-4 and Smad1, and phospho(p)Smad1/5/8 levels were assessed at different time points. Western blot analysis showed that ObI-1 over- expression significantly increases the activation of the Smad pathway (Fig. 7H, I). We can hypothesize that ObI-1 also acts on other proteins involved in BMPs signaling.

In addition, we observed that BMP signaling inhibition counteracts ObI-1-mediated stimulation of osteogenic differentiation. Indeed, treatment with the BMPR-I antagonist LDN193189 during osteogenic differentiation of mMSCs transfected with ObI-1 Flag completely abolished ObI-1mediated enhancement of differentiation (Fig. 7J-O and Supplementary Fig. S7B).

Collectively, these findings suggest that ObI-1 regulates osteogenic differentiation, at least in part, by stimulating BMP-4 expression and function. 

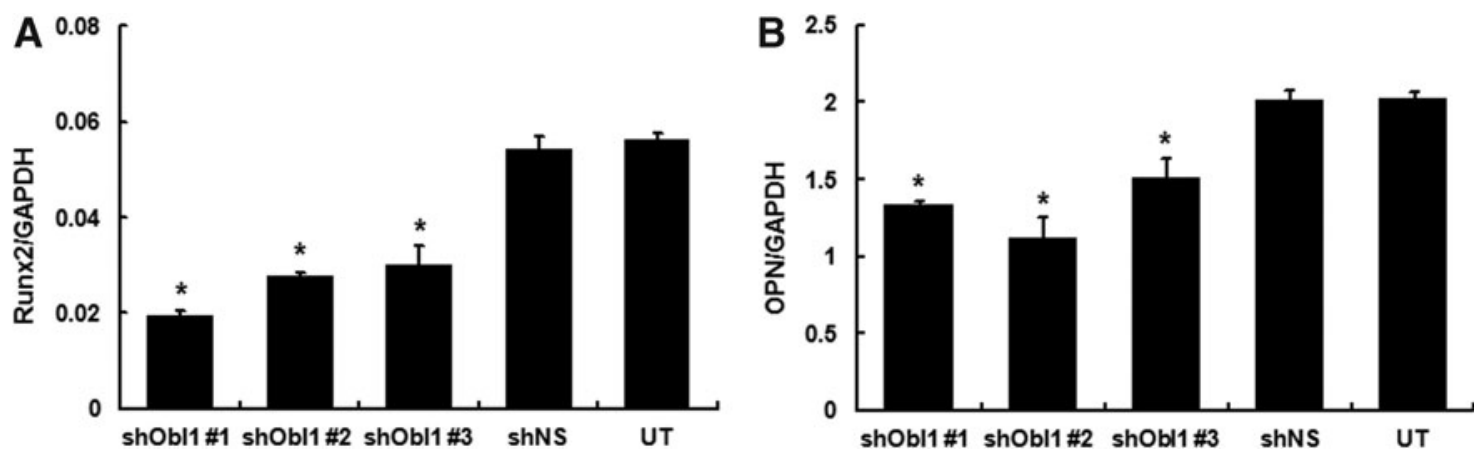

FIG. 5. ObI-1 silencing reduces the expression of osteogenic differentiation markers. Real-time PCR analysis was performed to evaluate expression profile of early osteogenic differentiation markers in W20-17 cells and reveals a significant decrease in (A) Runx2 and (B) OPN transcript levels in ObI-1-silenced cells compared with untransfected (UT) cells and cells transfected with shNS. Runx2 and OPN levels were evaluated at day 4 and 7 of differentiation, respectively. Results are expressed as Runx2/GAPDH and OPN/GAPDH levels ratio $(* P<0.05)$. Runx2, Runt-related transcription factor 2; OPN, osteopontin.

\section{Discussion}

Despite intensive investigation, our knowledge of the molecular mechanisms driving MSCs differentiation toward the osteogenic lineage is still incomplete. A better comprehension of this biological process is of crucial importance since bone-related pathologies constitute an increasing health care burden in industrialized countries and require safer and more effective therapeutic strategies [39]. MSCs represent a promising cell source for regenerative medicine applications
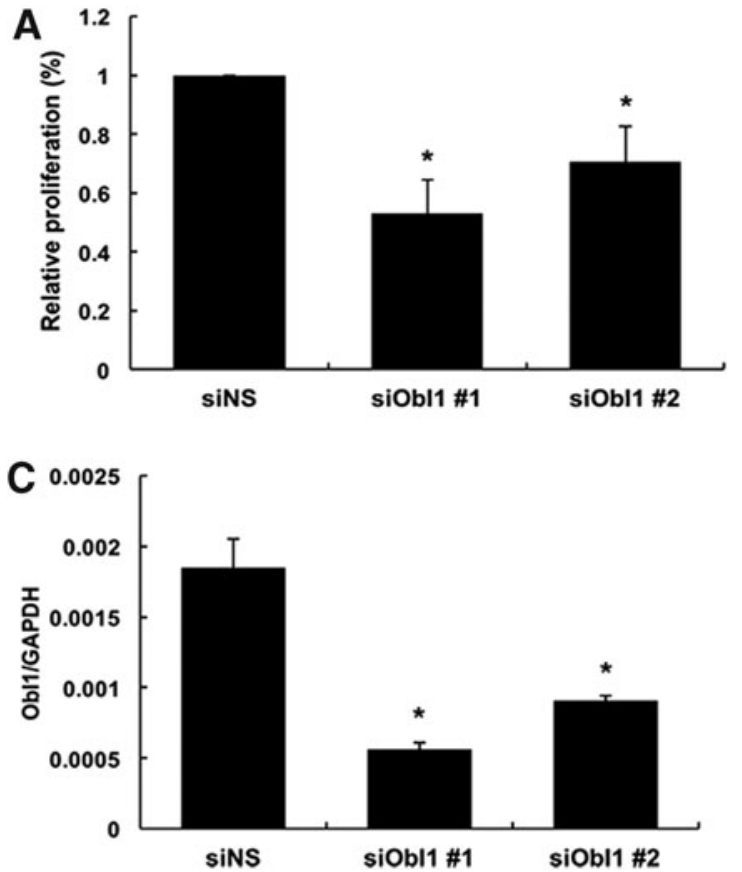

and an excellent model to study pathways relevant in osteoblast differentiation. These cells, indeed, present several advantages over other adult stem cells: they can be extensively expanded in vitro; they are immune-privileged and able to produce trophic factors [40] and several anti-inflammatory and immunomodulatory cytokines $[8,41,42]$. Therefore, the identification of the molecular bases of osteogenic differentiation is mandatory to regulate and optimize the process and to fully exploit MSC therapeutic potential.

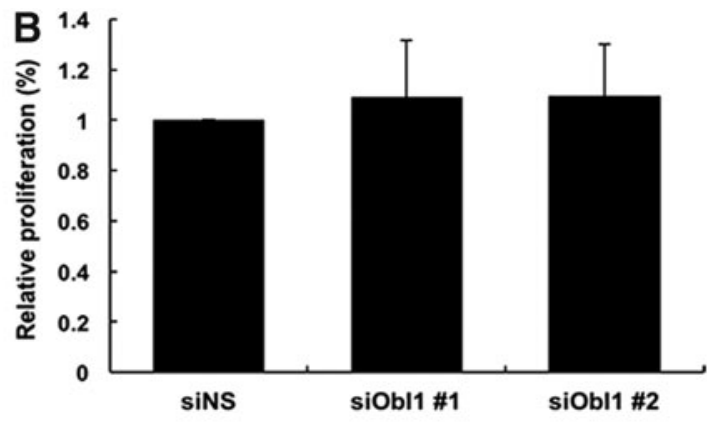

FIG. 6. ObI-1 silencing selectively impairs osteoprogenitors proliferation. mMSCs were transfected with either siNS or siRNAs targeting ObI-1 (siObI1\#1 or siObI-1\#2). (A, B) BrdU incorporation assay was performed to evaluate the proliferation of cells transfected with the siRNAs for $24 \mathrm{~h}$ and then either stimulated with osteogenic medium, preosteoblasts, (A) or cultured in regular media (undifferentiated mMSCs), (B) for the following $24 \mathrm{~h}$ ( $48 \mathrm{~h}$ post-transfection). ObI-1 silencing significantly decreases osteoprogenitor proliferation (A), while does not alter mMSC proliferation (B). Cell proliferation is calculated as the percentage of BrdU- over DAPI-positive nuclei and expressed as fold change of the proliferation of control cells (shNS), and was determined by counting 30 fields per condition $(10 \times$ magnification, $* P<0.01)$. (C) Silencing efficacy was confirmed by real-time PCR analysis $48 \mathrm{~h}$ after siRNA transfection: ObI-1 transcript levels are significantly reduced in mMSCs transfected with siObI1\#1 or siObI1\#2 compared with cells transfected with siNS. Results are expressed as ObI-1/ GAPDH levels ratio. siRNA, small interfering RNA; siNS, non-silencing siRNA; BrdU, bromo-2'-deoxyuridine. 

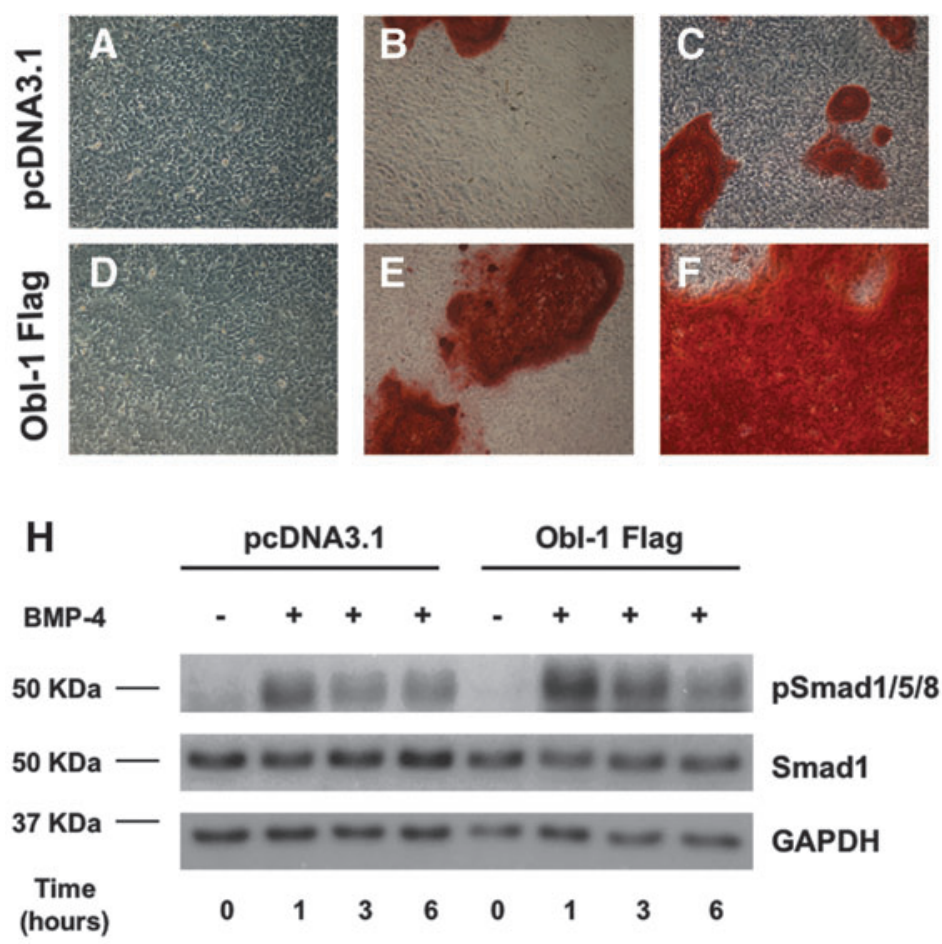

pcDNA3.1
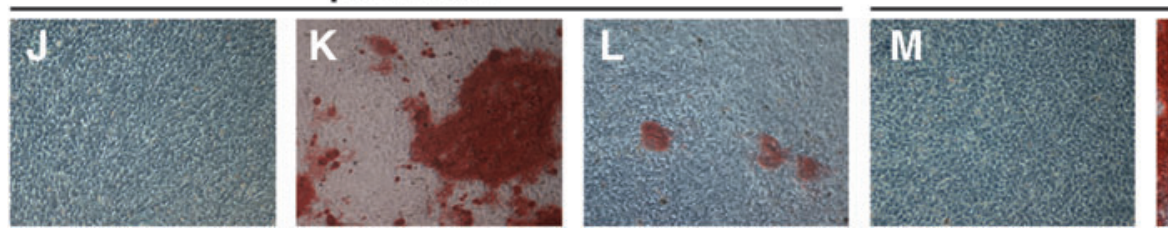

Obl-1 Flag

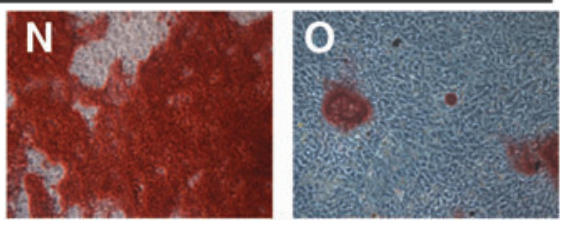

FIG. 7. ObI-1 modulates osteoblastogenesis through a BMP-mediated pathway. ObI-1 overexpression increases osteoblastmediated calcium deposition after 14 days of differentiation. mMSCs stably transfected with (A-C) pcDNA3.1 or (D, E) ObI-1 Flag were cultured for 2 weeks in $(\mathbf{A}, \mathbf{D})$ regular medium, $(\mathbf{B}, \mathbf{E})$ medium supplemented with ascorbic acid 2-phosphate, and glycerol 2-phosphate or $(\mathbf{C}, \mathbf{F})$ complete osteogenic medium. Alizarin red staining shows that ObI-1 overexpression enhances matrix mineralization compared with controls. Magnification $10 \times$. Pictures are representative of three independent experiments. (G) Real-time PCR analysis shows that BMP-4 transcript levels are significantly increased in mMSCs transfected with ObI-1 Flag compared with control cells. Results are expressed as BMP-4/GAPDH levels ratio $(* * P<0.01)$. (H) ObI-1 expression potentiates BMP-mediated Smad1/5/8 activation (phosphorylation). mMSCs stably transfected with either ObI-1 Flag or empty pcDNA3.1 were treated with $40 \mathrm{ng} / \mathrm{mL}$ BMP-4 and total Smad1 and phosphorylated (p)Smad1/5/8 levels were evaluated at different time points by western blot analysis. As controls, cells were maintained in medium only. ObI-1 Flag transfected cells show increased levels of pSmad1/5/8. GAPDH levels were included as loading control. (I) Densitometric analysis of the immunoreactive bands confirms an increased phosphorylation of Smads in cells transfected with ObI-1 Flag (white bars) compared with empty vector (black bars); results are reported as fold change of $\mathrm{pSmad} 1 / 5 / 8$ levels (previously normalized to Smad1 levels) in BMP4-stimulated cells versus untreated cells $(* P<0.05)$. mMSCs transfected with $(\mathbf{J}-\mathbf{L})$ pcDNA3.1 or $(\mathbf{M}-\mathbf{O})$ ObI-1 Flag were cultured for 2 weeks in $(\mathbf{J}, \mathbf{M})$ regular medium, $(\mathbf{K}, \mathbf{N})$ osteogenic medium alone, or $(\mathbf{L}, \mathbf{O})$ supplemented with $100 \mathrm{nM}$ of the BMPR-I antagonist LDN193189. Alizarin red staining shows that BMP inhibition strongly reduces matrix mineralization and abolishes ObI-1-mediated stimulation of differentiation. Magnification $10 \times$. Pictures are representative of three independent experiments. BMP, bone morphogenetic protein; BMPR-I, BMP receptor type I.

The present report focuses on the characterization of ObI-1, a novel putative transcriptional regulator of osteogenic differentiation. ObI-1 belongs to the family of KRAB/zinc-finger transcription factors, the largest family of transcriptional regulators in mammals. These transcription factors are present exclusively in tetrapod vertebrates and are involved in the regulation of several biological processes, such as cell proliferation and differentiation (including osteogenic), apoptosis, and neoplastic transformation $[28,43]$. The role of ObI-1 as transcription factor is also supported by its nuclear localization. Our finding is also consistent with a previous article that describes the same gene as a transcriptional repressor in mouse gonads [20].
As most regulatory proteins, ObI-1 levels and function seem to be tightly regulated by Ub-mediated proteasomal degradation. Proteasomal degradation, in addition to its role in protein turnover and degradation of misfolded products, plays an important regulatory role in fine-tuning the activity of proteins involved in a myriad of processes, from cell cycle regulation to DNA repair [44]. Since gene expression needs to be finely modulated in time and space and in response to external signals, it is not surprising that transcription factors and chromatin-modifying proteins constitute major targets for the Ub-proteasome system [34,45].

Moreover, most transcriptional factors, in particular those regulating cell growth and proliferation, are highly unstable 
proteins targeted by the Ub-proteasome system [46]. The proteasomal degradation of ObI-1, as well as its very short half-life, could indicate the requirement of tightly regulated levels of this protein within cells. Although we could not detect the ObI-1 protein by western blot analysis in stably transfected mMSCs, we were clearly able to appreciate the positive effects of its overexpression on osteogenic differentiation in ObI-1 transfected cells compare with the control. This could indicate that this protein is rapidly degraded after stabilization in response to a stimulus or undergoes cycles of stabilization and destabilization, hampering our ability to detect it. Further studies are required to elucidate the mechanisms regulating ObI-1 stability, the E3 ubiquitin ligases and deubiquitinating enzymes involved, as well as the specific stimuli inducing its stabilization.

Our data suggest that ObI- 1 is required in the early steps of osteoblast differentiation: in fact, its silencing impairs osteoprogenitors proliferation resulting in a reduction of both ALP expressing mature osteoblasts and mineral matrix deposition. Conversely, its overexpression enhances the differentiation process.

Osteoblast differentiation requires the early expression of the transcription factor Runx2, considered the master gene of bone formation [9,10]; this gene is expressed early during mouse embryonic development in the cells prefiguring the future skeleton and is able to regulate the expression of most bone-specific proteins [14]. Runx2 protein levels are also tightly regulated at transcriptional and translational levels as well as through posttranslational modifications, including phosphorylation, ubiquitination, and acetylation [47]. We observed that ObI-1 knockdown strongly reduces Runx 2 early expression, while its overexpression increases it; these data suggest that ObI-1 role in osteoblastogenesis is partly mediated by Runx 2 and that ObI- 1 may directly or indirectly regulate Runx 2 levels.

To identify a possible mechanism of action of ObI-1, we decided to evaluate whether BMPs-mediated signaling was involved. BMPs play a crucial role in bone development; these growth factors, locally synthesized by the skeleton, play an autocrine role in osteoblast differentiation acting on a large number of pathways and cellular functions [5,37]. Among other genes, BMPs are able to induce Runx2 expression that is essential to activate osteoblast differentiation, and Runx2-Smads cooperation is believed to mediate the activity of BMPs in modulating gene transcription in cells of the osteoblastic lineage $[17,38,48]$.

Our data suggest that ObI-1 role in osteogenic differentiation is, at least in part, mediated by the BMPs pathway. The following lines of evidence support this hypothesis: (1) endogenous ObI-1 silencing reduces BMP-4 transcript levels, whereas its over-expression increases them, respectively; (2) ObI-1 overexpression enhances BMP-4 stimulation of osteoblast differentiation increasing Smad1/5/8 phosphorylation; and (3) treatment with a BMPR-I antagonist completely abrogates ObI-1-mediated stimulation of osteogenic differentiation. BMPs act through pathways that involve several players and are subject to multiple types of regulation [37,49]; therefore, additional studies will be required to better characterized ObI-1 role and mechanism of action in modulating BMP signaling.

Despite their name, BMPs expression and function are not limited to bone. In fact, these proteins constitute a group of morphogens with pivotal roles during embryonic develop- ment and organogenesis as well as in adult tissue homeostasis [50]. These multifunctional growth factors regulate proliferation, differentiation, and function of a wide variety of cell types and are involved in processes as diversified as fracture repair, vascular remodeling, spermatogenesis, and iron metabolism [51-54]. We observed that ObI-1 is expressed in several mouse adult tissues and organs suggesting additional roles besides osteogenic regulation. ObI- 1 is expressed at high levels in lung, and the significance of this finding is currently uncharacterized. However, BMP signaling has been implicated in the regulation of lung morphogenesis, lung stem cell differentiation, adult lung homeostasis, and tissue-injury repair [55-58]. Therefore, it is tempting to speculate that ObI-1 could modulate BMPs effects in this organ.

According to bioinformatic analysis, ZNF717 is the best candidate as ObI-1 ortholog in humans. ZNF717 is present in humans as well as in other nonhuman primates and encodes a KRAB zinc-finger transcription factor. Our preliminary evaluation is consistent with the hypothesis that ZNF717 may be the ObI-1 human ortholog; ZNF717 is expressed in hMSCs and its levels increase during osteogenic differentiation with an expression profile comparable to what observed for ObI-1 in mMSCs. Furthermore, Håkelien et al. performed the first genome-wide analysis of Runx2 target genes in human osteoblasts. Interestingly, chromatin immunoprecipitation-seq analysis revealed that ZNF717 transcription seems to be regulated by Runx2 [59]. However, further studies are required to confirm the role of ZNF717 in human osteogenic differentiation as well as to elucidate its mechanisms of action and a possible modulation of BMP signaling. Recent studies also indicate that ZNF717 is recurrently altered in gastric tumors and hepatocellular carcinomas, and may be regulated by methylation [60-62].

In conclusion, we have identified a novel transcriptional regulator of osteogenic differentiation able to stimulate MSC commitment toward the osteogenic lineage and osteoblast maturation partly through BMP-mediated activation of Runx2. Further studies will be required to fully elucidate ObI-1 mechanism of action in osteoblast development and other biological processes.

\section{Acknowledgments}

We thank Daniela Sarnataro for technical help and assistance with confocal microscopy. This work was supported by the MIUR (grant no. PRIN prot. N. 2010B5B2NL_004).

\section{Author Disclosure Statement}

No competing financial interests exist.

\section{Supplementary Material}

Supplementary Figure S1 Supplementary Figure S2 Supplementary Figure S3 Supplementary Figure S4 Supplementary Figure S5 Supplementary Figure S6 Supplementary Figure S7 Supplementary Figure S8 Supplementary Figure S9 


\section{References}

1. Komori T. (2006). Regulation of osteoblast differentiation by transcription factors. J Cell Biochem 99:1233-1239.

2. Karsenty G, HM Kronenberg and C Settembre. Genetic control of bone formation. Annu Rev Cell Dev Biol 25:629-648.

3. Urist MR. (1965). Bone: formation by autoinduction. Science 150:893-899.

4. Wang RN, J Green, Z Wang, Y Deng, M Qiao, M Peabody, Q Zhang, J Ye, Z Yan, et al. (2014). Bone morphogenetic protein (BMP) signaling in development and human diseases. Genes Dis 1:87-105.

5. Chen G, C Deng and YP Li. (2012). TGF- $\beta$ and BMP signaling in osteoblast differentiation and bone formation. Int J Biol Sci 8:272-288.

6. Suzawa M, Y Takeuchi, S Fukumoto, S Kato, N Ueno, K Miyazono, T Matsumoto and T Fujita. (1999). Extracellular matrix-associated bone morphogenetic proteins are essential for differentiation of murine osteoblastic cells in vitro. Endocrinology 140:2125-2133.

7. Xiao G, R Gopalakrishnan, D Jiang, E Reith, MD Benson and RT Franceschi. (2002). Bone morphogenetic proteins, extracellular matrix, and mitogen-activated protein kinase signaling pathways are required for osteoblast-specific gene expression and differentiation in MC3T3-E1 cells. J Bone Miner Res 17:101-110.

8. Almalki SG and DK Agrawal. (2016). Key transcription factors in the differentiation of mesenchymal stem cells. Differentiation 92:41-51.

9. Ducy P, R Zhang, V Geoffroy, AL Ridall and G Karsenty. (1997). Osf2/Cbfa1: a transcriptional activator of osteoblast differentiation. Cell 89:747-754.

10. Komori T, H Yagi, S Nomura, A Yamaguchi, K Sasaki, K Deguchi, Y Shimizu, RT Bronson, YH Gao, et al. (1997). Targeted disruption of Cbfa1 results in a complete lack of bone formation owing to maturational arrest of osteoblasts. Cell 89: 755-764.

11. Ito Y, SC Bae and LS Chuang. (2015). The RUNX family: developmental regulators in cancer. Nat Rev Cancer 15:81-95.

12. Otto F, AP Thornell, T Crompton, A Denzel, KC Gilmour, IR Rosewell, GW Stamp, RS Beddington, S Mundlos, et al. (1997). Cbfa1, a candidate gene for cleidocranial dysplasia syndrome, is essential for osteoblast differentiation and bone development. Cell 89:765-771.

13. Lee B, K Thirunavukkarasu, L Zhou, L Pastore, A Baldini, J Hecht, V Geoffroy, P Ducy and G Karsenty. (1997). Missense mutations abolishing DNA binding of the osteoblast-specific transcription factor OSF2/CBFA1 in cleidocranial dysplasia. Nat Genet 16:307-310.

14. Ducy P, M Starbuck, M Priemel, J Shen, G Pinero, V Geoffroy, M Amling and G Karsenty. (1999). A Cbfa1dependent genetic pathway controls bone formation beyond embryonic development. Genes Dev 13:1025-1036.

15. Grafe I, S Alexander, JR Peterson, TN Snider, B Levi, B Lee and Y Mishina. (2017). TGF- $\beta$ family signaling in mesenchymal differentiation. Cold Spring Harb Perspect Biol 10: pii:a022202.

16. Lee KS, SH Hong and SC Bae. (2002). Both the Smad and p38 MAPK pathways play a crucial role in Runx 2 expression following induction by transforming growth factor-beta and bone morphogenetic protein. Oncogene 21:7156-7163.

17. Wu M, G Chen and YP Li. (2016). TGF-beta and BMP signaling in osteoblast, skeletal development, and bone formation, homeostasis and disease. Bone Res 4:16009.
18. Fitzsimmons REB, MS Mazurek, A Soos and CA Simmons. (2018). Mesenchymal stromal/stem cells in regenerative medicine and tissue engineering. Stem Cells Int 2018:8031718.

19. Querques F, B Cantilena, C Cozzolino, MT Esposito, F Passaro, S Parisi, B Lombardo, T Russo and L Pastore. (2015). Angiotensin receptor I stimulates osteoprogenitor proliferation through TGF $\beta$-mediated signaling. J Cell Physiol 230:1466-1474.

20. Jin S, H Choi, JT Kwon, J Kim, J Jeong, J Kim, S Ham, BN Cho, YJ Yoo and C Cho. (2015). Identification and characterization of reproductive KRAB-ZF genes in mice. Gene 565:45-55.

21. Esposito MT, R Di Noto, P Mirabelli, M Gorrese, S Parisi, D Montanaro, L Del Vecchio and L Pastore. (2009). Culture conditions allow selection of different mesenchymal progenitors from adult mouse bone marrow. Tissue Eng Part A 15:2525-2536.

22. Mariotti E, P Mirabelli, G Abate, M Schiattarella, P Martinelli, G Fortunato, R Di Noto and L Del Vecchio. (2008). Comparative characteristics of mesenchymal stem cells from human bone marrow and placenta: CD10, CD49d, and CD56 make a difference. Stem Cells Dev 17:1039-1041.

23. Boergermann JH, J, Kopf, PB Yu and P Knaus. (2010). Dorsomorphin and LDN-193189 inhibit BMP-mediated Smad, p38 and Akt signalling in $\mathrm{C} 2 \mathrm{C} 12$ cells. Int J Biochem Cell Biol 42:1802-1807.

24. Lee YC, CJ Cheng, MA Bilen, JF Lu, RL Satcher, LY YuLee, GE Gallick, SN Maity and SH Lin. (2011). BMP4 promotes prostate tumor growth in bone through osteogenesis. Cancer Res 71:5194-5203.

25. Kamitani T, K Kito, HP Nguyen and ET Yeh. (1997). Characterization of NEDD8, a developmentally down-regulated ubiquitin-like protein. J Biol Chem 272:28557-28562.

26. Parisi S, F Passaro, L Aloia, I Manabe, R Nagai, L Pastore and T Russo. (2008). Klf5 is involved in self-renewal of mouse embryonic stem cells. J Cell Sci 121:2629-2634.

27. Aloia L, S Parisi, L Fusco, L Pastore and T Russo. (2010). Differentiation of embryonic stem cell 1 (Dies1) is a component of bone morphogenetic protein 4 (BMP4) signaling pathway required for proper differentiation of mouse embryonic stem cells. J Biol Chem 285:7776-7783.

28. Urrutia R. (2003). KRAB-containing zinc-finger repressor proteins. Genome Biol 4:231.

29. Amable PR, MV Teixeira, RB Carias, JM Granjeiro and R Borojevic. (2013). Identification of appropriate reference genes for human mesenchymal cells during expansion and differentiation. PLoS One 8:e73792.

30. Quiroz FG, OM Posada, D Gallego-Perez, N Higuita-Castro, C Sarassa, DJ Hansford, P Agudelo-Florez and LE López. (2010). Housekeeping gene stability influences the quantification of osteogenic markers during stem cell differentiation to the osteogenic lineage. Cytotechnology 62:109-120.

31. Vandesompele J, K De Preter, F Pattyn, B Poppe, N Van Roy, A De Paepe and F Speleman. (2002). Accurate normalization of real-time quantitative RT-PCR data by geometric averaging of multiple internal control genes. Genome Biol 3:RESEARCH0034.

32. Pryszcz LP, J Huerta-Cepas and T Gabaldón. (2011). MetaPhOrs: orthology and paralogy predictions from multiple phylogenetic evidence using a consistency-based confidence score. Nucleic Acids Res 39:e32.

33. Filtz TM, WK Vogel and M Leid. (2014). Regulation of transcription factor activity by interconnected posttranslational modifications. Trends Pharmacol Sci 35: 76-85. 
34. Hammond-Martel I, H Yu and B Affar el. (2012). Roles of ubiquitin signaling in transcription regulation. Cell Signal 24:410-421.

35. Bellows CG, JE Aubin and JNM Heersche. (1991). Initiation and progression of mineralization of bone nodules formed in vitro: the role of alkaline phosphatase and organic phosphate. Bone 14:27-40.

36. Chen J, K Singh, BB Mukherjee and J Sodek. (1993). Developmental expression of osteopontin (OPN) mRNA in rat tissues: evidence for a role for OPN in bone formation and resorption. Matrix 13:113-123.

37. Canalis E, AN Economides and E Gazzerro. (2003). Bone morphogenetic proteins, their antagonists, and the skeleton. Endocr Rev 24:218-235.

38. Lee K-S, H-J Kim, Q-L Li, X-Z Chi, C Ueta, T Komori, JM Wozney, E-G Kim, J-Y Choi, H-M Ryoo and S-C Bae. (2000). Runx2 is a common target of transforming growth factor beta1 and bone morphogenetic protein 2, and cooperation between Runx 2 and Smad5 induces osteoblastspecific gene expression in the pluripotent mesenchymal precursor cell line C2C12. Mol Cell Biol 20:8783-8792.

39. Cauley JA. (2013). Public health impact of osteoporosis. J Gerontol A Biol Sci Med Sci 68:1243-1251.

40. Balestrieri ML, SJ Lu, F De Nigris, A Giovane, S Williams-Ignarro, FP D'Armiento, Q Feng, C Fiorito, G Testa, et al. (2010). Therapeutic angiogenesis in diabetic apolipoprotein E-deficient mice using bone marrow cells, functional hemangioblasts and metabolic intervention. Atherosclerosis 209:403-414.

41. Bruder SP, N Jaiswal and SE Haynesworth. (1997). Growth kinetics, self-renewal, and the osteogenic potential of purified human mesenchymal stem cells during extensive subcultivation and following cryopreservation. J Cell Biochem 64:278-294.

42. Nauta AJ and WE Fibbe. (2007). Immunomodulatory properties of mesenchymal stromal cells. Blood 110:3499-3506.

43. Jheon AH, B Ganss, S Cheifetz and J Sodek. (2001). Characterization of a novel $\mathrm{KRAB} / \mathrm{C} 2 \mathrm{H} 2$ zinc finger transcription factor involved in bone development. J Biol Chem 276:18282-18289.

44. Kirkin V and I Dikic. (2007). Role of ubiquitin- and Ubl-binding proteins in cell signaling. Curr Opin Cell Biol 19:199-205.

45. Muratani M and WP Tansey. (2003). How the ubiquitinproteasome system controls transcription. Nat Rev Mol Cell Biol 4:192-201.

46. Conaway RC, CS Brower and JW Conaway. (2002). Emerging roles of ubiquitin in transcription regulation. Science 17:1254-1258.

47. Jonason JH, G Xiao, M Zhang, L Xing and D Chen. (2009). Post-translational regulation of Runx 2 in bone and cartilage. J Dent Res 88:693-703.

48. Zaidi SK, AJ Sullivan, AJ van Wijnen, JL Stein, GS Stein and JB Lian. (2002). Integration of Runx and Smad regulatory signals at transcriptionally active subnuclear sites. Proc Natl Acad Sci U S A 99:8048-8053.

49. Brazil DP, RH Church, S Surae, C Godson and F Martin. (2015). BMP signalling: agony and antagony in the family. Trends Cell Biol 25:249-264.

50. Wagner DO, C Sieber, R Bhushan, JH Borgermann, D Graf and P Knaus. (2010). BMPs: from bone to body morphogenetic proteins. Sci Signal 3:mr1.

51. Tsuji K, A Bandyopadhyay, BD Harfe, K Cox, S Kakar, L Gerstenfeld, T Einhorn, CJ Tabin and V Rosen. (2006). BMP2 activity, although dispensable for bone formation, is required for the initiation of fracture healing. Nat Genet 38:1424-1429.

52. Huang Z, D Wang, K Ihida-Stansbury, PL Jones and JF Martin. (2009). Defective pulmonary vascular remodeling in Smad8 mutant mice. Hum Mol Genet 18:2791-2801.

53. Puglisi R, M Montanari, P Chiarella, M Stefanini and C Boitani. (2004). Regulatory role of BMP2 and BMP7 in spermatogonia and Sertoli cell proliferation in the immature mouse. Eur J Endocrinol 151:511-520.

54. Babitt JL, FW Huang, DM Wrighting, Y Xia, Y Sidis, TA Samad, JA Campagna, RT Chung, AL Schneyer, et al. (2006). Bone morphogenetic protein signaling by hemojuvelin regulates hepcidin expression. Nat Genet 38:531539.

55. Weaver M, JM Yingling, NR Dunn, S Bellusci and BL Hogan. (1999). Bmp signaling regulates proximal-distal differentiation of endoderm in mouse lung development. Development 126:4005-4015.

56. Lee JH, DH Bhang, A Beede, TL Huang, BR Stripp, KD Bloch, AJ Wagers, YH Tseng, S Ryeom, CF Kim, et al. (2014). Lung stem cell differentiation in mice directed by endothelial cells via a BMP4-NFATc1-thrombospondin-1 axis. Cell 156:440-455.

57. Rosendahl A, E Pardali, M Speletas, P Ten Dijke, CH Heldin and P Sideras. (2002). Activation of bone morphogenetic protein/Smad signaling in bronchial epithelial cells during airway inflammation. Am J Respir Cell Mol Biol 27:160-169.

58. Song Y, L Coleman, J Shi, H Beppu, K Sato, K Walsh, J Loscalzo and YY Zhang. (2008). Inflammation, endothelial injury, and persistent pulmonary hypertension in heterozygous BMPR2-mutant mice. Am J Physiol Heart Circ Physiol 295:H677-H690.

59. Håkelien AM, JC Bryne, KG Harstad, S Lorenz, J Paulsen, J Sun, TS Mikkelsen, O Myklebost and LA Meza-Zepeda. (2014). The regulatory landscape of osteogenic differentiation. Stem Cells 32:2780-2793.

60. Cui J, Y Yin, Q Ma, G Wang, V Olman, Y Zhang, WC Chou, CS Hong, C Zhang, et al. (2015). Comprehensive characterization of the genomic alterations in human gastric cancer. Int J Cancer 137:86-95.

61. Duan M, J Hao, S Cui, DL Worthley, S Zhang, Z Wang, J Shi, L Liu, X Wang, et al. (2018). Diverse modes of clonal evolution in HBV-related hepatocellular carcinoma revealed by single-cell genome sequencing. Cell Res 28:359-373.

62. Lando M, CS Fjeldbo, SM Wilting, BC Snoek, EK Aarnes, MF Forsberg, GB Kristensen, RD Steenbergen and H Lyng. (2015). Interplay between promoter methylation and chromosomal loss in gene silencing at 3p11-p14 in cervical cancer. Epigenetics 10:970-980.

Address correspondence to:

Lucio Pastore, $M D, P h D$

CEINGE-Biotecnologie Avanzate

Via Gaetano Salvatore, 486

Naples 80145

Italy

E-mail: lucio.pastore@unina.it

Received for publication July 24, 2018

Accepted after revision January 17, 2019

Prepublished on Liebert Instant Online January 18, 2019 